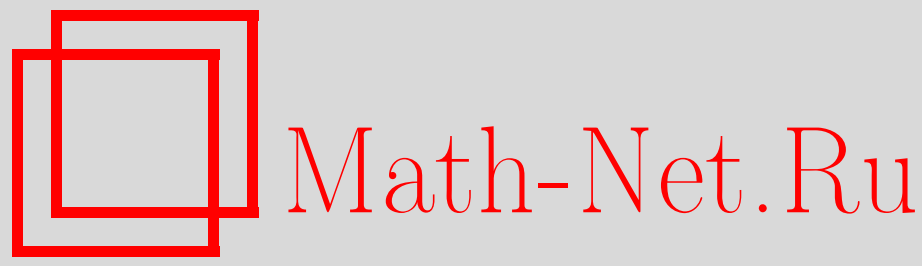

В. П. Маслов, Фазовые переходы в реальных газах и идеальные бозе-газы, ТМФ, 2011, том 167, номер 2, 295310

DOI: https://doi.org/10.4213/tmf6641

Использование Общероссийского математического портала Math-Net.Ru подразумевает, что вы прочитали и согласны с пользовательским соглашением http://www.mathnet.ru/rus/agreement

Параметры загрузки:

IP : 54.224 .60 .19

26 апреля 2023 г., $07: 22: 25$

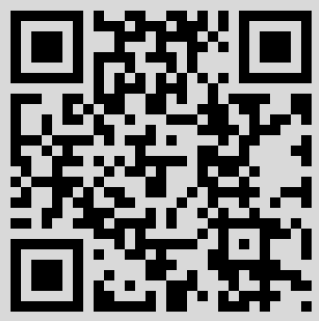


Том 167, № 2

май, 2011

(C) 2011 г.

В. П. Маслов*

\title{
ФАЗОВЫЕ ПЕРЕХОДЫ В РЕАЛЬНЫХ ГАЗАХ И ИДЕАЛЬНЫЕ БОЗЕ-ГАЗЫ
}

\begin{abstract}
Приводится новая концепция газа без учета взаимодействия частиц, основанная на теории чисел, в которой присутствуют фазовые переходы первого рода при $T<T_{\text {сr }}$ на изотермах. Приводятся формулы для новых идеальных газов, решающие парадокс Гиббса, а также формулы перехода к реальным газам, основанные на концепции Zeno line.
\end{abstract}

Ключевые слова: фазовые переходы первого рода, фазовые переходы второго рода, парадокс Эйнштейна, смеси газов, кластеры, идеальный газ, бозе-газ.

\section{1. ВВЕДЕНИЕ}

Настоящая статья является продолжением работы [1].

Согласно общепринятым курсам термодинамики идеальный газ - это газ, удовлетворяющий соотношению

$$
P V=R T
$$

где $P$ - давление, $V$ - объем, $T$ - температура, $R$ - газовая постоянная. Иначе говоря,

$$
\frac{P V}{R T}=1
$$

Величина $P V / R T$ называется фактором сжимаемости и обозначается числом $Z$. Это соотношение хорошо выполняется и для реальных газов при комнатных условиях, в том числе для смесей газов. А почему отношение $P V / R T$ равно именно единице? Так условились для удобства, подобрав соответствующим образом газовую постоянную.

Распределение Больцмана приводит к этому выражению для любых газов. Однако здесь возникает так называемый парадокс Гиббса. Если ответ, полученный из распределения Больцмана, поделить на $1 / N$ !, где $N$ - число частиц, то парадокс Гиббса в его исходной формулировке разрешается. Он разрешается для газа, полученного из молекул одного и того же вида. При этом необходимо сказать, что

Московский государственный университет им. М. В. Ломоносова, Москва, Россия. E-mail: v.p.maslov@mail.ru 
частицы этого газа неразличимы. Для смеси газов соотношение (1) все равно выполняется, а деление на $1 / N$ ! уже явно не годится, так как газы различимы.

А если это разные изотопы одного и того же вещества? Если два смешанных между собой газа совершенно идентичны, то мы должны делить на $1 /\left(N_{1}+N_{2}\right)$ !. А что делать, если они немного различаются? Получается все тот же скачок, что и в исходном парадоксе Гиббса с перегородкой между двумя газами. Кстати, решение исходного парадокса предлагалось в виде полупроницаемой перегородки (см., например, [2]-[5]). Наконец, если это бозе-газы с различными массами и мы их смешали? Этот парадокс, аналогичный парадоксу Гиббса, называется парадоксом Эйнштейна.

Из чисто математических теорем [6]-[18] автор получил, что для каждого отдельного газа одного типа молекул для невзаимодействующих частиц значение $Z$ не равно единице, а основную роль играет значение $Z_{\mathrm{cr}}$, т. е. фактор сжимаемости, выраженный через $T_{\mathrm{cr}}, P_{\mathrm{cr}}, \rho_{\mathrm{cr}}$, где $\rho_{\mathrm{cr}}-$ критическая плотность. Это связано с теорией чисел:

$$
Z_{\mathrm{cr}}=\frac{\zeta(\gamma+2)}{\zeta(\gamma+1)}
$$

где $\zeta(x)$ - дзета-функция Римана, $\gamma=d / 2, d$ - дробная размерность бозе-газа, который как квантовый реальный бозе-газ не имеет смысла, а может рассматриваться как задача диофантовых соотношений (7), (8) работы [1] (см. ниже).

Аналогия с теорией чисел позволяет решить проблему Ван-дер-Ваальса о точном вычислении "физической” энтропии. Именно проблемой вычисления энтропии Ван-дер-Ваальс в 1908 г. объяснял парадокс Гиббса. Он писал ([19], с. 201): “Сама энтропия не является непосредственно измеряемой величиной, и поэтому мы не можем ожидать разъяснения парадокса Гиббса при помощи измерений".

В работе [1] введен потенциал $\Omega_{\gamma}$ для теории чисел. Согласно физическим представлениям энтропия определяется как его производная по температуре. Непосредственным подсчетом можно показать, что она совпадает с энтропией Хартли для числа решений соотношений (7), (8) работы [1] (см., например, [20]).

Вопрос о смеси газов до сих пор остается актуальным. Для новых идеальных газов при $\mu \rightarrow-\infty$ ( $\mu$ - химический потенциал) выполняется соотношение $(1)$. Для их смесей это соотношение тоже имеет место. В отличие от новых идеальных газов, для реальных газов известно два типа смесей: гомогенная смесь и гетерогенная смесь. Гомогенная смесь - это такая смесь, в которой процентный состав газов не меняется. Примером такой смеси является воздух. Смесь воздуха и водяного пара гомогенна в очень узкой области температур, пока в паре не возникли в достаточно большом количестве водяные кластеры. Тогда смесь становится гетерогенной. Внутри капелек тумана исходный процентный состав газов меняется - смесь становится гетерогенной. Мы будем говорить только о таких смесях, в которых область гомогенности захватывает обе критические точки двух образующих компонент.

Кроме того, мы будем предполагать, что в этой области "идеальная кривая" (закон Бачинского) выполнена, т. е. кривая $Z=1$ на диаграмме $(\rho, T)$ является отрезком прямой Zeno line. Мы можем ее формально продолжить вплоть до точек 
$T=T_{\mathrm{B}}$ (температура Бойля) и $\rho=\rho_{\mathrm{B}}$ (плотность Бойля). Эти точки реально могут никогда не достигаться. Мы их определяем для удобства расчетов.

Экспериментальные данные [21] говорят о том, что для смеси двух газов возникает и средняя Zeno line. Предложенный в этой работе метод не дает возможности ее вычислить, хотя приведенное автором решение задачи рассеяния [1] связывает $Z_{\text {сr }}$ и Zeno line. Возможен способ выбора однопараметрического семейства потенциалов типа потенциала Леннарда-Джонса при разных значениях параметров, связывающих однозначно различные значения $Z_{\text {cr }}$ и Zeno line. Автор считает, что решение такой задачи облегчается тем, что для получения $Z_{\text {cr }}$ и Zeno line с помощью потенциала типа Леннарда-Джонса используется лишь скелет задачи (см. [1]), т. е. набор пар точек, отвечающих точкам покоя, а не функционал от потенциала взаимодействия.

Таким образом, после того как мы получаем идеальный (“чистый”) газ, отвечающий сумме чистых газов, мы можем применить приведенные ниже формулы перехода от идеального газа к реальному.

ЗАмечание 1. Стандартная нормировка в термодинамике $N / V \rightarrow$ const при $N \rightarrow \infty$ здесь заменяется на формулировку типа формулировки Ван-дер-Ваальса: $T_{\text {red }}=T / T_{\text {cr }}$, где $T_{\text {cr }}$ - критическая температура в точке спинодали жидкой фазы. Эта нормировка существенно меняет понятие термодинамического предела статистической физики, поскольку в идеальном бозе-газе имеются соотношения (см. (7), (8) в работе [1]), являющиеся диофантовыми соотношениями теории чисел, где $N \rightarrow \infty$ и $\mathcal{E} \rightarrow \infty$. Отсюда следует $\beta=1 / T \rightarrow 0$. Нормировка $T_{\text {red }}=T / T_{\text {cr }}$ заменяет "уравновешивание" $N$ в правой части соотношения (8) [1] с помощью объема $V$ и оставляет задачу в рамках чистой теории чисел. Отличие от формулировки Ван-дер-Ваальса состоит только в том, что $T_{\text {red }}=1$ в точке спинодали жидкой фазы. В идеальном газе температура этой точки определяется однозначно по значению $Z_{\mathrm{cr}}$.

\section{2. ПЕРЕХОД ОТ ИДЕАЛЬНОГО ГАЗА К РЕАЛЬНОМУ В ГАЗОВОЙ, ФЛЮИДНОЙ И ЖИДКОЙ ФАЗАХ}

По существу, реальный газ отличается от нового понятия идеального газа, введенного автором в [1], только тем, что линия $Z=1$ в идеальном газе отвечает значению $\mu=-\infty$, а в реальном газе достигается при $\mu>-\infty$. При этом поведения плотностей температуры и давления при $Z=1$ связаны совершенно определенными соотношениями. На плоскости $(\rho, T)$ это наклонная линия, называемая Zeno line (см. [1]). Иначе говоря, достаточно знания констант $T_{\mathrm{B}}$ и $\rho_{\mathrm{B}}$.

Если значение $\gamma=\gamma_{0}$ отвечает $Z_{\text {cr }}$, в нашем распределении (37) из работы [1] происходит умножение на функцию от $V$, т. е. следующая замена в распределении Бозе-Эйнштейна:

$$
V \rightarrow \varphi_{\gamma}(V), \quad \frac{\varphi_{\gamma}(V)}{V} \rightarrow \text { const } \quad \text { при } \quad V \rightarrow \infty .
$$

Константа зависит от температуры (от точки на Zeno line) и находится из краевой задачи для каждого $T$. При $T=1$ константа равна единице. Эта функция постоянна при $\gamma \geqslant \gamma_{0}$, где $\gamma_{0}=0.2$ есть критическая размерность, отвечающая значению 
$Z_{\text {cr }}=0.29[1]$. Поэтому величина $Z$, получаемая при $\mu=0$, имеет вид

$$
\widetilde{Z}=\frac{V \varphi_{\gamma_{0}}^{\prime}(V)}{\varphi_{\gamma_{0}}(V)} \cdot \frac{\zeta\left(\gamma_{0}+2\right)}{\zeta\left(\gamma_{0}+1\right)}=0.29 .
$$

В газовой фазе при $\gamma \geqslant \gamma_{0}$ константа в (4) равна единице. Для нового идеального газа, чтобы получить значение

$$
\frac{P_{\mathrm{cr}} V_{\mathrm{cr}}}{R T_{\mathrm{cr}}}=Z_{\mathrm{cr}}=\frac{\zeta\left(\gamma_{0}+2\right)}{\zeta\left(\gamma_{0}+1\right)},
$$

нужно взять $\gamma_{0}=0.222$.

Повторим подробнее выкладки работы [1].

Для любого $Z<1$ имеем

$$
\begin{aligned}
Z & =\frac{V \varphi_{\gamma_{0}}^{\prime}(V)}{\varphi_{\gamma_{0}}(V)} \cdot \frac{\Gamma\left(\gamma_{0}+1\right)}{\Gamma\left(\gamma_{0}+2\right)}\left(\int_{0}^{\infty} \frac{\varepsilon^{\gamma_{0}+1} d \varepsilon}{e^{\xi-\kappa}-1}\right)\left(\int_{0}^{\infty} \frac{\varepsilon^{\gamma_{0}} d \varepsilon}{e^{\xi-\kappa}-1}\right)^{-1}= \\
& =\frac{V \varphi_{\gamma_{0}}^{\prime}(V)}{\varphi_{\gamma_{0}}(V)} \Psi(\kappa), \quad \kappa=\frac{\mu}{T}, \quad \varphi_{\gamma_{0}}^{\prime}(V)=\frac{\partial \varphi}{\partial V}, \quad V=\frac{Z T}{P},
\end{aligned}
$$

где $\Gamma(\cdot)$ - гамма-функция. При $\kappa=0$ получаем (5). Далее мы находим $\mu(T, V)$ как функцию от $V$ из условия $Z=1$ :

$$
\frac{V \varphi_{\gamma_{0}}^{\prime}(V)}{\varphi_{\gamma_{0}}(V)} \Psi(\kappa)=1, \quad \kappa=\kappa(V) .
$$

С другой стороны, при $Z=1$ выполняется условие на Zeno line, следующее из еe наклона

$$
P=\rho_{\mathrm{B}} T\left(1-\frac{T}{T_{\mathrm{B}}}\right)
$$

или

$$
P=T_{\mathrm{B}} \rho\left(1-\frac{\rho}{\rho_{\mathrm{B}}}\right),
$$

а также

$$
T=T_{\mathrm{B}}\left(1-\frac{\rho}{\rho_{\mathrm{B}}}\right) .
$$

Следовательно, зависимости $P(T), T(\rho), P(\rho)$ известны: $P(T)$ - парабола Бачинского, $T(\rho)$ - прямая, $P(\rho)$ - парабола.

Найдем $P$ согласно распределению Бозе-Эйнштейна, где $V$ заменено на $\varphi_{\gamma_{0}}(V)$ :

$$
\begin{gathered}
P=\frac{\varphi_{\gamma_{0}}^{\prime}(V) T^{\gamma_{0}+2}}{\Gamma\left(\gamma_{0}+2\right)} \int_{0}^{\infty} \frac{\varepsilon^{\gamma_{0}+1} d \varepsilon}{e^{-\kappa} e^{\varepsilon}-1}, \\
\varphi_{\gamma_{0}}^{\prime}(V) \operatorname{Li}_{\gamma_{0}+2}(y)=\frac{\rho}{T_{\mathrm{B}}^{\gamma_{0}+1}\left(1-\rho / \rho_{\mathrm{B}}\right)^{\gamma_{0}+1}}, \quad \rho=\frac{1}{V}, \\
\frac{V \varphi_{\gamma_{0}}^{\prime}(V)}{\varphi_{\gamma_{0}}(V)} \frac{\operatorname{Li}_{\gamma_{0}+2}(y)}{\operatorname{Li}_{\gamma_{0}+1}(y)}=1,
\end{gathered}
$$


где $\mathrm{Li}$ - полилогарифм. Начальное условие для $\gamma>\gamma_{0}$ и $T<T_{\text {cr }}$ определяется из условия $\varphi_{\gamma_{0}}(V) / V \rightarrow 1$ при $V \rightarrow \infty$. Значение ${ }^{1)} \varphi_{\gamma_{0}}(V)$ не меняется при $\gamma>\gamma_{0}$.

При $T>T_{\text {cr }}$, также как и при $\gamma<\gamma_{0}$, начальное условие имеет вид

$$
\frac{\varphi(V)}{V} \rightarrow \text { const. }
$$

Эта константа выбирается так, чтобы изотерма идеального газа при $T<T_{\text {сr }}$ и при $Z=1$ попала бы на отвечающую ей точку $T$ на Zeno line. А при $T>T_{\text {cr }}$ и при $Z=1$ изохора, выпущенная из критической изотермы к плоскости $(Z, P)$, попала бы в соответствующую ей точку $\rho$ на Zeno line.

Естественно, что понятие нового идеального газа [1] должно обобщать понятие обычного идеального газа и при определенных значениях параметров (температуры, давления, плотности) переходить в него. Новый идеальный газ, связанный с теорией чисел, как бозе- и ферми-газы, при химическом потенциале, стремящемся к $-\infty$, должен переходить в старый идеальный газ, и мы получаем $Z=1$. Оказывается, что в силу выполнения условия на Zeno line (см. [1]) и экспериментального ее наблюдения, нужно дополнительно знать только одно число - отношение температуры Бойля к критической температуре, и с помощью достаточно простых преобразований (решения уравнения первого порядка и “подгонки" (методом стрельбы) его начального условия) можно получить из новых идеальных газов термодинамику любых реальных газов.

ЗАмечаниЕ 2. При нормировке $T_{\mathrm{cr}}=1, P_{\mathrm{cr}}=1$ получаем, что $\rho_{\mathrm{cr}}=1 / Z_{\mathrm{cr}}$. Вместе с тем на Zeno line

$$
\rho_{\mathrm{cr}}=\rho_{\mathrm{B}}\left(1-\frac{1}{T_{\mathrm{B}}}\right)
$$

Значит,

$$
1=Z_{\mathrm{cr}} \rho_{\mathrm{B}}\left(1-\frac{1}{T_{\mathrm{B}}}\right) .
$$

Отсюда на Zeno line получаем

$$
P=Z_{\text {cr }} \rho_{\mathrm{B}}\left(1-\frac{T}{T_{\mathrm{B}}}\right) T=Z_{\mathrm{cr}} T_{\mathrm{B}}\left(1-\frac{\rho}{\rho_{\mathrm{B}}}\right) \rho,
$$

если $T_{\mathrm{cr}}=1, P_{\text {cr }}=1$.

При $T<1$ в распределении Бозе-Эйнштейна размерности $\gamma_{0}$ объем $V$ заменяется на $\varphi_{\gamma_{0}}\left(\rho_{\mathrm{cr}} V\right)$. При $T>1$ объем $V$ заменяется на $\varphi_{\gamma}\left(\rho_{\mathrm{cr}} V\right)$, где $\gamma>\gamma_{0}$, причем начальное условие заменяется на краевое. Для идеального газа $T>T_{\text {cr }}$ (для флюидов) строятся изохоры-изодимы (постоянная плотность и постоянная размерность $\gamma$, от-

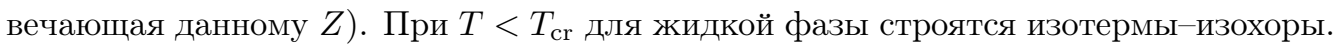

Замена начального условия на краевое для $\varphi_{\gamma}\left(\rho_{\mathrm{cr}} V\right)$ возникает и в случае жидкой фазы.

1) В статье [1] была допущена досадная опечатка: в формулах (26)-(29) пропущено умножение на объем $V$. Условие $\varphi_{\gamma}(V) / V \rightarrow 1$ при $V \rightarrow \infty$ не выполнимо без этого множителя. 


\section{3. НОРМИРОВКА НА ТЕМПЕРАТУРУ РТУТИ. РЕГУЛЯРИЗАЦИЯ И ПОПРАВКА К РАСПРЕДЕЛЕНИЮ БОЗЕ-ЭЙНШТЕЙНА}

Прежде всего произведем необходимую перенормировку распределения БозеЭйнштейна. Она заключается не в том, чтобы интеграл от распределения был равен единице. Такой интеграл может, вообе говоря, расходиться. Например, так происходит для распределения типа распределения Парето, если степень убывания распределения достаточно медленная.

Предположим, что распределение убывает как $1 / \ln (|x|+1)$. Даже его квадрат не интегрируем. С точки зрения гильбертовых пространств это обобщенная функция. Рассмотрим в качестве примера на интервале $(-\pi, \pi)$ функции $\left\{e^{i \pi n}\right\}$. Если коэффициенты разложения по этим функциям убывают как $1 / \ln (|x|+1)$, то в гильбертовом пространстве функций $L_{2}$ на отрезке $(-\pi, \pi)$ это обобщенная функция. Далее предположим, что ее максимум находится при $n=6 \cdot 10^{23}$. Это означает, что с точки зрения гильбертовых пространств $L_{2}$ они столь быстро осциллируют, что представляют собой обобщенную функцию, равную нулю на пространстве бесконечно дифференцируемых финитных функций с точностью до $10^{-23}$. Тем не менее мы нормируем на этот максимум и изучаем асимптотики относительно этого максимума, как бы перемещаясь в другой мир. Мы как бы берем в качестве начала отсчета (т. е. единицы) температуру в самых жарких тропиках, тропическую температуру.

Ван-дер-Ваальс, уже зная стандартную нормировку "терминологического предела" $N / V \rightarrow$ const, заметил новую, которая дала знаменитый закон соответственных состояний, позволяющий классифицировать газы. Главная нормировка $T=1$. Мы замечаем, что если в исходной статистической физике считать "термодинамическим пределом" именно это соотношение, то в исходной задаче теории чисел о диофантовых соотношениях (7), (8) работы [1] уже не нужно прибегать к введению дополнительного параметра $V$ (или попросту положить $V=1$ ), поскольку при этой замечательной нормировке $N$ становится конечным (см. ниже (13)) и может означать плотность $\rho$, а $1 / N$ - "удельный объем".

В бозе-газе $T_{\text {сr }}$ есть температура вырождения, она находится как величина, стремящаяся к бесконечности. Фактически это соответствует нормировке распределения на его максимум, очень удаленный от нуля градусов по Кельвину. Число частиц, при котором образуется конденсат при такой нормировке, равно

$$
N_{\mathrm{cr}}=\Gamma\left(\gamma_{0}+1\right) \zeta\left(\gamma_{0}+1\right)
$$

и, следовательно, не очень велико.

Для $\gamma \leqslant 0$ необходимо провести поправку к распределению Бозе-Эйнштейна, а именно воспользоваться формулой (37) работы [1] $]^{2)}$. Найдем асимптотику соотношения

$$
N=\int_{0}^{\infty} t^{\gamma} d t\left[\frac{1}{\left(e^{t} / z\right)-1}-\frac{N}{\left(e^{t N} / z^{N}\right)-1}\right]=\Gamma(\gamma+1)\left[\operatorname{Li}_{\gamma+1}\left(e^{-\xi}\right)-\frac{\operatorname{Li}_{\gamma+1}\left(e^{-N \xi}\right)}{N^{\gamma}}\right]
$$

в точке $N_{\text {cr }}(13)$.

2)Этот член получается из парастатистики, поскольку число частиц на каждом уровне энергии не может быть больше $N$ - числа частиц в газе. С другой стороны, $N$ достаточно велико, чтобы можно было в парастатистике воспользоваться оценками Эйлера-Маклорена [22]. 
Соотношение (14) находится вблизи точки (13) и уточняет формулу Бозе-Эйнштейна.

Отметим, что градуировка Кельвина неопределенна, поскольку абсолютного нуля достичь невозможно. Вообще, более естественно для градуирования Кельвина рассматривать $\ln T$. Тогда ноль переходит в минус бесконечность, которую достичь невозможно, а 1 градус Кельвина переходит в ноль. Недостижимость минус бесконечности более наглядна, чем невозможность достижения нуля.

Тогда безразлично, от какой точки нужно вести отсчет. В силу этого $\ln T$ определяется с точностью до константы, а значит, $T$ определяется с точностью до умножения на константу. Поэтому невозможно определить $T_{\text {cr }}$ для одного газа в каких-либо единицах (например, по Кельвину). Поэтому наиболее естественно для идеального газа рассматривать нормировку на $T_{\text {cr }}$, так как, например, градуировка по Цельсию связана с реальным газом $\mathrm{H}_{2} \mathrm{O}$ [23], [24].

Таким образом, для решения парадокса Эйнштейна мы должны были учесть “фрактальные” дробные размерности, к которым приводит смесь двух бозе-эйнштейновских газов, у которых имеются две разные температуры вырождения $T_{1}$ и $T_{2}$ (пусть $T_{1}>T_{2}$ ), зависящие от процентного соотношения объемов газов. В силу приводимых ниже уравнений мы находим температуру $T_{\mathrm{cr}}^{\mathrm{sum}}$ и соответствующую ей размерность $\gamma_{0}^{\text {sum }}$. А поскольку мы учитываем неразличимость частиц при подсчете их плотности, то это уточнение приводит и к решению парадокса Гиббса.

Если мы рассматриваем всю шкалу газов, то обнаруживаем, что значения $Z_{\text {сr }}$ ограничены сверху. Самое большое значение $Z_{\text {cr }}$ у ртути (0.39). Близкое значе-

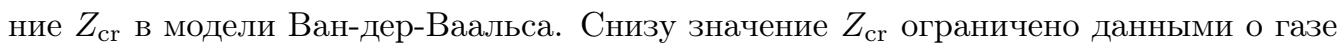
$\mathrm{He}^{3}$, для которого существенным образом проявляются квазиклассические эффекты (см. [1]). Поэтому нормировку для шкалы газов нужно провести, взяв $T / T_{\text {cr }}$ для ртути. Остальные газы должны подстраиваться под эту нормировку.

Для отдельного газа нормировка $T_{\mathrm{cr}}=1$ и $P_{\mathrm{cr}}=1$ возможна. Но если мы сравниваем разные газы и изучаем их гомогенные смеси, то наиболее естественно рассмат-

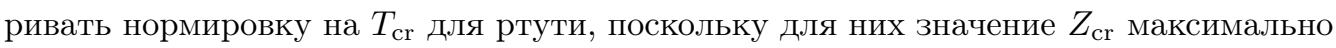
среди всех газов. В этом случае для классического газа размерности $\gamma$ критическая температура будет числом, меньшим 1:

$$
T_{\mathrm{cr}}^{\gamma}=\left(\frac{\zeta\left(\gamma_{0}+2\right)}{\zeta(\gamma+2)}\right)^{1 /(\gamma+2)}
$$

где $\gamma_{0}=0.33$ для ртути. Затем мы находим отвечающую ей точку спинодали жидкой фазы и перенормируем на нее.

\section{4. НОВЫЙ КЛАССИЧЕСКИЙ ИДЕАЛЬНЫЙ ГАЗ КАК АЛЬТЕРНАТИВА БОЗЕ-КОНДЕНСАТУ}

Все решения диофантовых уравнений (7), (8) в работе [1] мы считали равновероятными. Как мы показывали на примере распределения $\mathcal{E}$ купюр по $N$ зрителям, если $N>N_{\text {cr }}$, то $N-N_{\text {сr }}$ зрителей окажутся без купюр. При этом, разумеется, имелось в виду, что $N / N_{\mathrm{cr}} \geqslant 1+\delta$, где $\delta>0-$ любое фиксированное число, 
а $N \rightarrow \infty, \mathcal{E} \rightarrow \infty$ и $\mathcal{E} \gg N$. Этот факт, как мы уже неоднократно писали, соответствует бозе-конденсату в физике, а в экономике может определять, например, число безработных.

Альтернативой этому явлению в теории чисел является объединение при $N=$ $2 N_{\text {cr }}$ в пары, а при увеличении $N$ - в кластеры. Объединение означает, как уже отмечалось ранее, уменьшение размерности, что соответствует уменьшению степеней свободы членов объединения.

Возникает вопрос: как объединяться оптимальным образом, т.е. как наилучшим образом уменьшить размерность? Уменьшение размерности (связанное с "числом степеней свободы") имеет место в экономике как за счет объединения, так и за счет ограничения "рыночной свободы" законами. Как выбрать наилучший путь?

В приводимом неоднократно в работах автора примере с Коровьевым ${ }^{3)}$ говорилось, что достаточно зрителям объединиться в группы по 10 человек, и тогда каждая группа с огромной вероятностью получит по меньшей мере одну купюру, члены группы поделят деньги, и никто не умрет с голоду. Эта процедура приведет к значительному падению энтропии, так как в каждой группе раздача добытых денег будет идти по некоторому порядку, например всем поровну. Но можно вообще всем объединиться и “честно" поделить весь миллион, который раздает Коровьев. При таком варианте энтропия падает до нуля. И в том, и в другом случае главная задача будет выполнена - все зрители останутся живы, в термодинамике это означает, что число частиц сохранится. Задача заключается, однако, в том, чтобы число зрителей (частиц) сохранилось при том, что число людей в объединении было бы минимальным. Это означает, что нужно получить максимальную размерность при сохранении числа частиц. Этот переход к максимальной размерности мы будем называть переходом в жидкое состояние. Если для газовой ветки изотермы мы брали размерность $\gamma=\gamma_{0}$ и не меняли это значение при $\gamma>\gamma_{0}$, то в жидкой ветке при понижении температуры понижается и значение $\gamma$, а следовательно, и значение $Z_{\mathrm{cr}}^{\gamma}$.

Очевидно, что при уменьшении температуры число частиц в объединении, т. е. число молекул в кластерах, возрастает. Чтобы получить это возрастание, мы должны повысить исходную температуру (при $\gamma=\gamma_{0}$ ) для энтропии, при этом исходное число решений диофантовых уравнений увеличится. Тогда число молекул объединения (кластера) будет больше. А это означает, что в исходной формуле для энтропии мы должны увеличить $T_{\mathrm{cr}}$. Таким образом, при уменьшении реальной температуры жидкой фазы мы должны увеличить относительную температуру $T_{\text {cr }}$ в исходной формуле для энтропии. Это означает, что относительная температура при $Z^{\gamma_{0}}$ увеличивается и исходная энтропия принимает вид

$$
S=\left(\frac{T}{T_{\text {liq }}}\right)^{\gamma+1}\left(\operatorname{Li}_{\gamma+2}\left(e^{-\xi}\right)(\gamma+2)+\xi \operatorname{Li}_{\gamma+1}\left(e^{-\xi}\right)\right),
$$

где $\xi=-\mu / T$ (см. [18]).

ЗАмЕчАНИЕ 3. Полилогарифм $\operatorname{Li}_{\gamma+2}\left(e^{-\xi}\right)$ имеет вид

$$
\operatorname{Li}_{\gamma+2}\left(e^{-\xi}\right)=\sum \frac{e^{-k \xi}}{k^{\gamma+2}}=\sum \frac{e^{-k \xi-\gamma \ln k}}{k^{2}} .
$$

3) Коровьев в варьете показывал фокус: разбрасывал 1000000 купюр над 10000 зрителей. 
Производная по $\gamma$ от него равна

$$
\frac{\partial}{\partial \gamma} \operatorname{Li}_{\gamma+2}\left(e^{-\xi}\right)=-\sum \frac{\ln k e^{-k \xi-\gamma \ln k}}{k^{2}} .
$$

При $\mu=0$ получаем

$$
\left.S\right|_{\mu=0}=\left(\frac{T}{T_{\text {liq }}}\right)^{\gamma+1} \zeta(\gamma+2)(\gamma+2) .
$$

Отсюда возникает простое условие для максимизации $\gamma$ :

$$
\begin{gathered}
\left.\frac{d S}{d \gamma}\right|_{\xi=0}=0, \quad \ln \left(\frac{T}{T_{\text {liq }}}\right) \zeta(\gamma+2)(\gamma+2)+\zeta_{\gamma}^{\prime}(\gamma+2)(\gamma+2)+\zeta(\gamma+2)=0, \\
-\frac{d \ln \zeta(\gamma+2)}{d \gamma}=\ln \left(\frac{1}{T_{\text {liq }}}\right)+\frac{1}{\gamma+2} .
\end{gathered}
$$

Для ртути, как для са́мого высокого значения $Z_{\mathrm{cr}}$ для всех реальных газов, мы полагаем $T_{\text {cr }}=1$. Тогда решение (18) дает $\gamma=0.14$ и, следовательно, $Z_{\gamma_{1}\left(\gamma_{0}\right)}=0.24$.

Поскольку $N=$ const, следовательно, на изотерме

$$
P_{\gamma_{1}}=\text { const } \cdot Z_{\gamma_{1}} \text {. }
$$

Точки $Z_{\gamma_{1}}, P_{\gamma_{1}}$ являются точками спинодали для жидкой фазы, т. е. точками окончания метастабильного состояния жидкой фазы.

ЗАмЕчАНиЕ 4. Метод градиентного спуска по энтропии описывает неустойчивую часть, и его траектория в эксперименте не наблюдается. Однако, чтобы графики не были разрывными для газовой и жидкой фазы, более привычно решать уравнение градиентного спуска от $\gamma_{0}$ до $\gamma_{1}\left(\gamma_{0}\right)$ от точки $\gamma=\gamma_{0}, \xi=0$ вплоть до точки $\gamma_{1}=0.14$.

Для ртути мы должны положить $T_{\text {red }}=T / T_{\text {cr }}$, т. е. $T_{\text {red }}=1$ при $T=T_{\text {cr. }}$ Если $T<T_{\text {cr }}$, то $T_{\text {red }}^{\text {liq }}=T / T_{\text {cr. }}$.

Поскольку в каждой точке жидкости при $\gamma<\gamma_{1}$ температура меньше $T_{\text {cr }}$, но каждой точке соответствует свое значение $Z_{\mathrm{cr}}^{\gamma}$, удобно нормировать каждое значение $Z_{\text {cr }}^{\gamma}$ на отвечающее ему значение $T_{\text {liq }}^{\gamma}$, что означает

$$
T_{\text {red }}=\frac{1}{T_{\mathrm{cr}}},
$$

т. е. увеличение $T_{\mathrm{cr}}^{\gamma_{0}}$, а следовательно, и энтропии $S$, на новое $T_{\mathrm{cr}}$, paвное $1 / T_{\text {red }}$, жидкости

$$
S_{\gamma_{0}}=T_{\mathrm{cr}}^{\gamma+1}\left(\operatorname{Li}_{\gamma_{0}+2}\left(e^{-\xi}\right)\left(\gamma_{0}+2\right)+\xi \operatorname{Li}_{\gamma_{0}+1}\left(e^{-\xi}\right)\right) .
$$

Таким образом, в формулах (18), (19) полагаем $T=1 / T_{\text {liq }}$.

Будем называть газ квазиидеальным, если $\varphi_{\gamma}^{\prime}(V)=$ const (см. [18]). В этом случаe Zeno line участвует в определении точек спинодалей жидкой фазы, но жидкость остается несжимаемой. Только учет функции $\varphi_{\gamma}(V)$ позволяет изогнуть прямые и учесть сжимаемость жидкости. Однако при $T_{\text {liq }} \ll 1$, т. е. при достаточно малых 
(но еще положительных) $\gamma$ значение $\varphi_{\gamma}(V)$ уже сказывается несущественно. Поэтому квазиидеальный газ и особенно смесь квазиидеальных газов дают достаточно хорошее приближение спинодалей жидкой фазы.

Геометрическое место точек квазиидеальных спинодалей ${ }^{4)}$ задается формулой

$$
P=T \rho_{\mathrm{B}}\left(1-\frac{T}{T_{\mathrm{B}}}\right) Z_{\mathrm{cr}}^{\gamma},
$$

где $\rho_{\mathrm{B}}$ и $T_{\mathrm{B}}-$ плотность Бойля и температура Бойля соответственно. Здесь

$$
Z_{\mathrm{cr}}^{\gamma\left(T_{\text {liq }}\right)}=\frac{\zeta\left(2+\gamma\left(T_{\text {liq }}\right)\right)}{\zeta\left(1+\gamma\left(T_{\text {liq }}\right)\right)}, \quad T=T_{\text {liq }} .
$$

Напомним, что $\gamma\left(T_{\text {liq }}\right)$ вычисляется из алгебраического соотношения (19).

Каждая точка на квазиидеальной спинодали соединяется с соответствующей заданному значению $\gamma\left(T_{\text {liq }}\right)$ точкой на Zeno line левее критической прямой при $\gamma\left(T_{\text {liq }}\right)=1$. При $\gamma(T)<0$ мы используем поправку к распределению Бозе-Эйнштейна, введенную автором.

Найдем константы $b$ и $\kappa$ из следующих соотношений:

$$
\begin{gathered}
\int_{0}^{\infty} \xi\left\{\frac{1}{e^{b(\xi+\kappa)}-1}-\frac{N}{e^{b k(\xi+\kappa-1)}}\right\} \xi^{\gamma} d \xi=\mathcal{E}, \\
\int_{0}^{\infty}\left\{\frac{1}{e^{b(\xi+\kappa)}-1}-\frac{N}{e^{b k(\xi+\kappa-1)}}\right\} \xi^{\gamma} d \xi=N,
\end{gathered}
$$

где $\kappa=-\mu, b=1 / T$.

Обозначим $n=\mathcal{E}$. При $\kappa=0$ имеем

$$
n=\int \frac{\xi d \xi^{\alpha}}{e^{b \xi}-1}=\frac{1}{b^{1+\alpha}} \int_{0}^{\infty} \frac{\eta d \eta^{\alpha}}{e^{\eta}-1},
$$

где $\alpha=\gamma+1$. Отсюда

$$
b=\frac{1}{n^{1 /(1+\alpha)}}\left(\int_{0}^{\infty} \frac{\xi d \xi^{\alpha}}{e^{\xi}-1}\right)^{1 /(1+\alpha)} .
$$

Обозначив $N_{\mathrm{cr}}=k_{0}$, получим

$$
\begin{aligned}
k_{0}= & \int_{0}^{\infty}\left\{\frac{1}{e^{b \xi}-1}-\frac{k_{0}}{e^{k_{0} b \eta}-1}\right\} d \xi^{\alpha}= \\
= & \frac{1}{b^{\alpha}} \int_{0}^{\infty}\left(\frac{1}{e^{\xi}-1}-\frac{1}{\xi}\right) d \xi^{\alpha}+\frac{1}{b^{\alpha}} \int_{0}^{\infty}\left(\frac{1}{\xi}-\frac{1}{\xi\left(1+\left(k_{0} / 2\right) \xi\right)}\right) d \xi^{\alpha}- \\
& -\frac{k_{0}^{1-\alpha}}{b^{\alpha}} \int_{0}^{\infty}\left\{\frac{k_{0}^{\alpha}}{e^{k_{0} \xi}-1}-\frac{k_{0}^{\alpha}}{k_{0} \xi\left(1+\left(k_{0} / 2\right) \xi\right)}\right\} d \xi^{\alpha} .
\end{aligned}
$$

\footnotetext{
4) Т. е. точек окончания метастабильного состояния жидкой фазы.
} 


\section{Положим}

$$
c=\int_{0}^{\infty}\left(\frac{1}{\xi}-\frac{1}{e^{\xi}-1}\right) \xi^{\gamma} d \xi
$$

После замены $k_{0} \xi=\eta$ получим

$$
\begin{aligned}
& \frac{k_{0}^{1-\alpha}}{b^{\alpha}} \int_{0}^{\infty}\left\{\frac{k_{0}^{\alpha}}{e^{\eta}-1}-\frac{k_{0}^{\alpha}}{\eta(1+\eta / 2)}\right\} d \xi^{\alpha}= \\
& =\frac{k_{0}^{1-\alpha}}{b^{\alpha}} \int_{0}^{\infty}\left\{\frac{1}{e^{\eta}-1}-\frac{1}{\eta(1+\eta / 2)}\right\} d \eta^{\alpha}= \\
& =\frac{k_{0}^{1-\alpha}}{b^{\alpha}}\left\{\int_{0}^{\infty}\left(\frac{1}{e^{\eta}-1}-\frac{1}{\eta}\right) d \eta^{\alpha}+\int_{0}^{\infty} \frac{d \eta^{\alpha}}{2(1+\eta / 2)}\right\}=-c \frac{k_{0}^{1-\alpha}}{b^{\alpha}}+c_{1} \frac{k_{0}^{1-\alpha}}{b^{\alpha}} .
\end{aligned}
$$

Поскольку

$$
\frac{1}{\eta(1+\eta / 2)}=\frac{1}{\eta}-\frac{1}{2(1+\eta / 2)},
$$

обозначив

$$
c_{1}=\int_{0}^{\infty} \frac{d \eta^{\alpha}}{2(1+\eta / 2)}
$$

мы можем написать

$$
\begin{aligned}
\int_{0}^{\infty}\left(\frac{1}{\xi}-\frac{1}{\xi\left(1+k_{0} \xi / 2\right)}\right) d \xi^{\alpha} & =\frac{k_{0}}{2} \int_{0}^{\infty} \frac{d \xi^{\alpha}}{1+k_{0} \xi / 2}= \\
& =\left(\frac{k_{0}}{2}\right)^{1-\alpha} \int_{0}^{\infty} \frac{d \eta^{\alpha}}{1+\eta}=c_{1}\left(\frac{k_{0}}{2}\right)^{1-\alpha}
\end{aligned}
$$

Следовательно,

$$
\begin{aligned}
k_{0}= & -\frac{1}{b^{\alpha}} c_{1}+\frac{1}{b^{\alpha}} c\left(\frac{k_{0}}{2}\right)^{1-\alpha}-\frac{k_{0}^{1-\alpha}}{b^{\alpha}} \int_{0}^{\infty}\left\{\frac{1}{e^{\eta}-1}-\frac{1}{\eta(1-\eta / 2)}\right\} d \eta^{\alpha}- \\
& -\frac{1}{2} \int \frac{d \eta^{\alpha}}{1+\eta / 2} \cdot \frac{k_{0}^{1-\alpha}}{b^{\alpha}}=\frac{1}{b^{\alpha}} c+\frac{k_{0}^{1-\alpha}}{b^{\alpha}} c .
\end{aligned}
$$

Поскольку $k_{0}$ - число частиц, $b=1 / T, \alpha=1+\gamma$, то $k_{0} b^{\alpha}$ при $\gamma>0$ есть функция Римана $\zeta(1+\gamma)$. Поэтому $k_{0}^{\gamma+1}$ при $\gamma<1$ растет, и первый член правой части уравнения (30) может быть отброшен. Полагая в этой асимптотике $T_{\mathrm{cr}}=1$, получим, что функцию

$$
\mathcal{M}(\gamma+1)=\left(\frac{c(\gamma)}{\Gamma(\gamma+1)}\right)^{1 /(1+\gamma)}
$$

естественно считать продолжением дзета-функции Римана в дополнительную область аргументов $0>\gamma \geqslant-1$.

6 Теоретическая и математическая физика, т. 167, № 2, 2011 г. 
ЗАмЕЧАниЕ 5. Асимптотика формулы (24) при $\mu<0$ может быть представлена в следующем виде:

$$
\int \frac{t^{\gamma} d t}{k_{2} e^{-\mu k_{1}} e^{t / k_{2}}-1}-\Gamma(\gamma+1) \operatorname{Li}_{\gamma+1}\left(e^{\mu}\right)
$$

что при $k_{1} \rightarrow \infty, k_{2} \rightarrow \infty, \mu \rightarrow 0$ переходит в $c(\gamma)$.

Фактор сжимаемости $Z_{\gamma}=-\zeta(\gamma+2) / \mathcal{M}(\gamma+1)$ испытывает скачок ${ }^{5)}$ от $\gamma=0$ до $\gamma<0$. Мы получаем окончание метастабильной области отрицательных давлений. Отсюда следует парадоксальный эффект увеличения плотности при уменьшении температуры, наблюдаемый в эксперименте (см. [26], а также видео на сайте http://www.iem.ac.ru//staff/kiril, где это явление наблюдается).

В рассматриваемом подходе мы получаем, что траектория удовлетворяет соотношению

$$
\mu d N=0
$$

Таким образом, новый идеальный газ представляет собой разрывные изотермы, состоящие из газовой ветви и несжимаемой жидкой ветви.

С точки зрения экономики это означает, что уменьшение "свободы" происходит вначале за счет объединения, а далее за счет ужесточения законов так, например, чтобы не было безработных и число $N$ сохранялось. С точки зрения физики это значит, что для $T>1$ при увеличении числа $N$ происходит каскадное увеличение объединений в кластеры, а затем возникает явление несжимаемости - увеличение давления не приводит к уменьшению объема.

Траектория, состоящая при $T>1$ из двух пересекающихся прямых на плоскости $(Z, P)$, следующая из теории чисел, является скелетом термодинамики и отвечает новому идеальному газу. Сглаживание (“обрастание мягкой тканью”) этого скелета дает учет взаимодействия.

ЗАмЕчАниЕ 6. Фазовый переход первого рода на плоскости $(P, Z)$ при $T<T_{\mathrm{cr}}$ происходит при $P=P_{\text {сr }}$ и изменении плотности и переменном химическом потенциале, а фазовый переход первого рода при $T<T_{\text {cr }}$ совершается на изотерме при разных давлениях и разных химических потенциалах. На диаграмме Ван-дер-Ваальса ему соответствует переход от газовой ветви к жидкой на всем интервале метастабильных состояний: от точки спинодали на газовой ветви до точки спинодали на жидкой ветви.

Чтобы получить фазовый переход стандартного вида при $T<T_{\mathrm{cr}}$, нужно найти на обеих изотермах точку, в которой у них равны химические потенциалы (правило Максвелла). Это отдельное условие проецирования на плоскость $(T, P)$ двух лагранжевых многообразий с краем (край отвечает спинодали), при котором потенциал Гиббса принимает максимальное значение. Иначе говоря, химический потенциал вдоль изотермы минимизируется.

\footnotetext{
5) Скачок спинодали при переходе через точку ноль от давлений положительных (сжатия) к давлениям отрицательным (растяжению) для жидкости является естественным, поскольку подобно резине с мелкими порами, в которой закон Гука на сжатие и растяжение меняется скачком, производная от давления по плотности также меняется скачком. Разумеется, для реального газа этот скачок сглажен [25].
} 
Так называемое геометрическое квантование этих двух многообразий может быть произведено туннельным каноническим оператором, где малым параметром служит $1 / N$. Это единственный малый параметр в теории чисел. Для реального газа согласно [1] естественно выбирать в качестве малого параметра диффузию газа (см. раздел 6).

\section{5. ПАРАДОКС ЭЙНШТЕЙНА И ИДЕАЛЬНЫЙ ГАЗ СМЕСИ}

Смесь идеальных газов в старом понимании идеального газа отвечает парадоксу Гиббса. Автор поступил радикально: отказался от старого понятия идеального газа и перешел к новому понятию идеального газа и, соответственно, к новому понятию смеси идеальных газов.

Описывая реальный газ, мы можем построить потенциал $\Omega$, несколько отличающийся от бозе-газа дробной размерности в газовой фазе и существенно отличающийся для флюидов и жидкой фазы [1].

Приведем формулировку парадокса бозе-статистик, который часто сопоставляют с парадоксом Гиббса [27]. Эйнштейн писал ([28], с. 481): “В заключение я хочу обратить внимание на один парадокс, который мне не удалось объяснить. С помощью изложенного здесь метода не представляет труда рассмотреть также случай смеси двух разных газов. В этом случае каждый сорт молекул имеет свои особые "ячейки". Отсюда следует аддитивность энтропии компонент смеси. Таким образом, каждая компонента в смысле энергии молекул, давления и статистического распределения ведет себя так, как будто в объеме смеси находится она одна. Смесь же $n_{1}$ молекул одного сорта и $n_{2}$ молекул другого, в которой молекулы первого и второго сорта отличаются друг от друга как угодно мало (особенно в отношении масс молекул $\left.m_{1}, m_{2}\right)$, при данной температуре имеет иное давление и иное распределение состояния, чем простой газ с числом молекул $n_{1}+n_{2}$, обладающий практически той же массой молекул и находящийся в том же объеме. Однако это представляется почти невозможным".

Если мы рассматриваем квантовый бозе-газ, для которого имеется симметрия по $n_{1}$ частицам первого типа и по $n_{2}$ частицам второго типа в начальной функции $\psi$, то решение очевидно. Наш гамильтониан не симметричен по перестановке $p_{i}^{(1)}, q_{i}^{(1)}$ и $p_{i}^{(2)}, q_{i}^{(2)}$, где верхний индекс означает первый и второй тип частиц. Даже если мы симметризуем начальную функцию $\psi$, то эта симметрия немедленно пропадет при $t>0$. Да и сама симметризация есть скачок оператора симметрии.

Другое дело, та статистика бозе-газа, которая, как мы видели в работе [1], отвечает задаче теории чисел. Если речь идет о вычислении точки бозе-конденсата, то вычисление размерности суммы отвечает, как это следует из [1], и бозе-конденсату в той же точке $Z_{\mathrm{cr}}^{\text {sum }}$. Следовательно, мы получим правило Эйнштейна о сложении бозе-газов любой дробной размерности с соответствующими значениями бозе-конденсатов (т.е. критических точек). Каждой массе $m_{1}$ и $m_{2}$ отвечают свои значения $T_{\mathrm{cr}}^{(1)}$ и $T_{\mathrm{cr}}^{(2)}$, или $N_{\mathrm{cr}}^{(1)}$ и $N_{\mathrm{cr}}^{(2)}$, согласно закону соответственных состояний

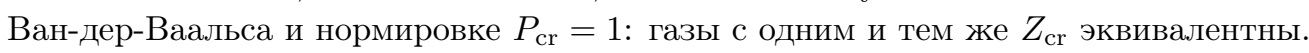

Мы говорим о смеси газов с разными значениями критических величин, если мы определяем плотность этой смеси. Это вновь сводится к задаче теории чисел и к 
диофантовым соотношениям. Наша задача ввести новое понятие - новый идеальный газ смеси газов. Из закона сохранения массы для $N_{\mathrm{cr}}^{\left(\gamma_{1}\right)}, N_{\mathrm{cr}}^{\left(\gamma_{2}\right)}$ и $N_{\mathrm{cr}}^{(\gamma)}$ следует, что

$$
\begin{gathered}
\left(\alpha m_{1}+\beta m_{2}\right)^{\gamma+1}\left(T_{\mathrm{cr}}^{(\gamma)}\right)^{\gamma+1} \zeta(\gamma+1)=\alpha m_{1}^{\gamma_{1}+1}\left(T_{\mathrm{cr}}^{\left(\gamma_{1}\right)}\right)^{\gamma_{1}+1} \zeta\left(\gamma_{1}+1\right)+ \\
+\beta m_{2}^{\gamma_{2}+1}\left(T_{\mathrm{cr}}^{\left(\gamma_{2}\right)}\right)^{\gamma_{2}+1} \zeta\left(\gamma_{2}+1\right), \quad \alpha+\beta=1 .
\end{gathered}
$$

Из аддитивности энтропии следует, что

$$
\begin{aligned}
& \left(\alpha m_{1}+\beta m_{2}\right)^{\gamma+1}(\gamma+2) \zeta(\gamma+2)\left(T_{\mathrm{cr}}^{(\gamma)}\right)^{\gamma+1}=m_{1}^{\gamma_{1}+1} \alpha\left(\gamma_{1}+2\right) \zeta\left(\gamma_{1}+2\right)\left(T_{\mathrm{cr}}^{\left(\gamma_{1}\right)}\right)^{\gamma_{1}+1}+ \\
& \quad+m_{2}^{\gamma_{2}+1} \beta\left(\gamma_{2}+2\right) \zeta\left(\gamma_{2}+2\right)\left(T_{\mathrm{cr}\left(\gamma_{2}\right)}\right)^{\gamma_{2}+1}
\end{aligned}
$$

(оба тождества справедливы в неравновесном состоянии).

Аналог Zeno line и функция $\varphi_{\gamma_{0}}(V)$ для смеси будут подробно исследованы в следующей статье.

\section{6. КРИТИЧЕСКИЕ ПОКАЗАТЕЛИ, ГИПОТЕЗА СКЕЙЛИНГА, ТУННЕЛЬНЫЙ КАНОНИЧЕСКИЙ ОПЕРАТОР И ПРАВИЛО МАКСВЕЛЛА}

Все рассуждения, как приведенные выше, так и основанные на потенциале взаимодействия Леннарда-Джонса, базируются на роли притяжения между частицами или, что то же самое, на возникновении объединений (димеров и кластеров) при определении нового идеального газа. Роль отталкивания была лишь вспомогательной, когда говорилось о значении малой вязкости для димера, попавшего в ямку-ловушку, а именно о том, что за счет малой вязкости димер опустится в конце концов на дно ямки.

Во введенном понятии идеального газа те состояния, которые являются метастабильными на жидкой и газовой ветвях идеального газа, ничем не отличаются от стабильного состояния, которое соответствует правилу Максвелла, при котором химические потенциалы на этих ветвях должны быть равны друг другу. Этот замечательный факт объясняется как раз отталкиванием, а значит, вязкостью. С этим же моментом, по мнению автора, связана и гипотеза Уиндома о скейлинге.

Поскольку данные классической теории критических показателей не совпали с экспериментальными данными и Уиндомом в 1965 г. была разработана другая гипотеза, в известной мере противоречащая основным постулатам термодинамики, то казалось бы естественным, что разрешение проблемы парадокса Гиббса должно ликвидировать и это противоречие. Действительно, в новой теории перехода от нового идеального газа к реальному возникает неаналитичность. А именно, производная функции $\varphi_{\gamma}(V)$ по $\gamma$ равна нулю при $\gamma \leqslant \gamma_{0}$ и $T<T_{\mathrm{cr}}$, где $\gamma_{0}$ отвечает критической точке, а далее при $\gamma>\gamma_{0}$ она отлична от нуля.

Однако независимость критических показателей, вычисленных согласно гипотезе скейлинга от шести критических значений газа, заставляет предположить, что главное противоречие заключается в некотором дополнительном моменте. Автору не известно экспериментальное подтверждение критических параметров для гомологической смеси, вычисленных на основе гипотезы скейлинга, но нет сомнений, что и они подтвердятся. 
После того, как мы построили строгую математическую концепцию термодинамики, необходимо дать ответ и на это несоответствие. В этом разделе мы дадим ответ на этот вопрос, хотя выкладки и численные совпадения еще не получены.

В молекулярной физике известна связь вязкости и диффузии. Параметр диффузии и параметр $i h$, где $h$ - постоянная Планка, тесно связаны (см., например, формулы для инстантонов в [29] и [30]). Автор также неоднократно писал, что так называемые термодинамические потенциалы в фазовом пространстве $T, P, V,-S$ образуют двумерную поверхность, названную автором [31] лагранжевым многообразием в фазовом пространстве $\left(q_{1}, q_{2}, p_{1}, p_{2}\right)$, а термодинамические потенциалы суть представление действия $\mathbb{S}=\int p d q$ в разных локальных картах этого многообразия.

Как сказано выше, термодинамика реального газа, построенная автором, не аналитична в критической точке. Однако главный член туннельного канонического оператора [32], где роль малого параметра играет диффузия, при максвелловском фазовом переходе характеризуется скачком газовой ветви в жидкую ветвь при одинаковом значении химического потенциала. Точно так же, как вязкость в термодинамике сглаживает ударную волну, диффузия "размывает" этот скачок плотности. Это, однако, не так заметно на эксперименте. Однако в критической точке, как в момент зарождения ударной волны (например, для уравнения Бюргерса), влияние канонического оператора и диффузии тем сильнее, чем больший фокус образуется. Поэтому вклад вблизи фокальной точки (в нашем случае - критической точки) дает существенную поправку к критическому индексу, полученному из асимптотики канонического оператора. Этот вклад не зависит от значения критической точки.

Универсальность, т. е. независимость от величины диффузии, а зависимость только от дефекта фокальной точки [33], связана с тем, что расстояние от критической точки есть также малый параметр, много меньший, чем параметр диффузии. Для простейших фокальных точек, выраженных через функцию Эйри или функцию Вебера, правило скейлинга выполняется. Дефект фокальной точки [33] есть инвариант, связанный с ее проецированием на координатную плоскость $T, P$. Аналогично проецирование на другие координатные плоскости дадут другие значения критических параметров.

Эта процедура в математике в случае осциллирующего не туннельного канонического оператора носит название "геометрического квантования" (см. [34]). Соответственно, операторы рождения димеров и других кластеров также обобщают понятие вторичного квантования, расширяют область применения термодинамики на полуклассический случай (см. [35]) и позволяют, в частности, получить сверхтекучесть классических газов (например, $\mathrm{H}_{2} \mathrm{O}$ ) в нанотрубках [36]-[38], которая подтверждена экспериментами.

Благодарности. Автор выражает глубокую благодарность В. С. Воробьеву за полезные обсуждения.

\section{Список литературы}

[1] В. П. Маслов, ТМФ, 165:3 (2010), 543-567.

[2] М. Планк, Термодинамика, ГИЗ, Л.-М., 1925.

[3] O. Wiedeburg, Ann. Phys., 289:12 (1894), 684-697. 
[4] A. Byk, Ztschr. Phys. Chem., 43/45 (1903), 465-495.

[5] Г. А. Лоренц, Лекиии по термодинамике, Гостехиздат, М., 1946.

[6] V.P. Maslov, Russ. J. Math. Phys., 18:1 (2011) (to appear).

[7] V.P. Maslov, Math. Notes, 86:3-4 (2009), 522-529.

[8] V.P. Maslov, Math. Notes, 86:5-6 (2009), 605-611.

[9] V.P. Maslov, Math. Notes, 87:3-4 (2010), 384-391.

[10] V.P. Maslov, Russ. J. Math. Phys., 17:2 (2010), 240-250.

[11] V.P. Maslov, Math. Notes, 87:5-6 (2010), 728-737.

[12] V.P. Maslov, J. Fixed Point Theory Appl., 8:1 (2010), 81-111.

[13] V.P. Maslov, Math. Notes, 88:1-2 (2010), 57-66.

[14] V.P. Maslov, Russ. J. Math. Phys., 17:3 (2010), 288-306.

[15] V.P. Maslov, Zeno-line, binodal, T- $\rho$ diagram and clusters as a new Bose-condensate bases on new global distributions in number theory, arXiv: 1007.4182.

[16] V.P. Maslov, Math. Notes, 88:3-4 (2010), 516-523.

[17] V.P. Maslov, Math. Notes, 88:5-6 (2010), 723-731.

[18] В. П. Маслов, Матем. заметки, 89:2 (2011), 272-284.

[19] Я. Ван-дер-Ваальс, Ф. Констамм, Курс термостатики, т. 1, ОНТИ, М., 1936.

[20] I. A. Molotkov, Russ. J. Math. Phys., 17:4 (2010), 476-485.

[21] В. В. Сычев, А. А. Вассерман, А. Д. Козлов, Г. А. Спиридонов, В. А. Цымарный, Термодинамические свойства воздуха, Изд-во стандартов, М., 1978.

[22] В. П. Маслов, В. Е. Назайкинский, Матем. заметки, 83:2 (2008), 232-263.

[23] В. П. Маслов, ТМФ, 160:2 (2009), 331-332.

[24] В.П. Маслов, Матем. заметки, 83:5 (2008), 787-791.

[25] V. P. Maslov, P. P. Mosolov, "Nonlinear Wave Equations Perturbed by Viscous Terms", De Gruyter Expositions in Mathematics, 31, Walter de Gruyter, Berlin, 2000.

[26] К. И. Шмулович, Л. Меркьюри, Вестник Отделения наук о Земле РАН, 1 (2006), 24.

[27] Я. М. Гельфер, В.Л. Любошиц, М. И. Подгорецкий, Парадокс Гиббса и тождественность частич в квантовой механике, Наука, М., 1975.

[28] А. Эйнштейн, Собрание научных трудов, т. 4: Квантовая теория одноатомного идеального газа, Наука, М., 1966.

[29] M. Dykman, L. Pryadko, Lectures of Theory of Dissipative Tunneling, http://www.pa.msu.edu/ dykman/PHY972/instanton_lectures.pdf.

[30] В. П. Маслов, "Глобальная экспоненциальная асимптотика решений туннельных уравнений и задачи о больших уклонениях", Международная конферениия по аналитическим методам в теории чисел и анализе (Москва, 14-19 сентября 1981 г.), Тр. МИАН CCCP, 163, 1984, 150-180.

[31] В. П. Маслов, Теория возмущений и асимптотические методы, Изд-во МГУ, М., 1965.

[32] V.P. Maslov, Russ. J. Math. Phys., 17:4 (2010), 454-467.

[33] В. П. Маслов, Асимптотические методы и теория возмущений, Наука, М., 1988.

[34] Н. Харт, Геометрическое квантование в действии, Мир, М., 1985.

[35] V.P. Maslov, Quantization of Thermodynamics and Ultrasecond Quantization, IKI Publishing House, M., 2001.

[36] V.P. Maslov, Russ. J. Math. Phys., 14:3 (2007), 304-318.

[37] V.P. Maslov, Russ. J. Math. Phys., 14:4 (2007), 453-464.

[38] V.P. Maslov, Russ. J. Math. Phys., 15:1 (2008), 98-101.

Поступила в редакцию 8.02.2011 